\title{
Optimal Control and Design Guidelines for Soft Jumping Robots: Series Elastic Actuation and Parallel Elastic Actuation in comparison
}

\author{
Riccardo Incaini, Leonardo Sestini, Manolo Garabini, Manuel Catalano, Giorgio Grioli and Antonio Bicchi
}

\begin{abstract}
A properly designed elastic actuation can increase the jumping height that a legged robot can reach.

In this paper we compare the two most popular conceptual soft actuator designs, parallel elastic (PEA) and series elastic (SEA), in the task of maximizing the jumping height. Such task is translated into an optimal control problem.

For a simplified version of the problem an analytical solution is provided, while a problem with more realistic constraints (e.g. the linear torque-speed motor characteristic is taken into account) is stated as a convex optimization problem and numerically solved.

The results show that: (i) given the power of the motor there exists an optimal constant stiffness that maximizes the performance for both the SEA and the PEA; (ii) the optimal stiffness depends on the task terminal time, the inertial parameters of the system and the reduction ratio of the motor; (iii) in the condition considered the SEA behaves better than the PEA.
\end{abstract}

\section{INTRODUCTION}

Since the presentation of the first robotic actuator composed of an electric motor and an elastic element connected in series (SEA [1]), soft actuators are becoming more and more popular in robotics. With respect to rigid actuation, they show better performance in terms of peak speed (see e.g. [2], [3], and [4]) and energy consumption in cyclic tasks (see e.g. [5], [6], and [7]).

An important field where the application of soft actuation is analyzed and exploited is legged locomotion, from devices with one degree of actuation (see e.g. [8], [9]) to fully actuated humanoids (see e.g. [10] and [11]).

On a conceptual level, literature presents mostly two design philosophies: SEA (in this category we find the robots presented in [10], [11] and [8]), and PEA (see e.g. [7]), in which a motor is connected in series or in parallel with an elastic element, respectively.

A comparison between these and other possible soft actuation schemes realizing ankle joints have been proposed by Grimmer et al. [12] where the analysis is performed in terms of energy consumption.

In this paper we analyze the task of maximizing the jumping height of two different robot designs, the PEA and the SEA. In doing this we take into account conceptual model for robotic structures employed in running or walking robots. This is not the case of jumping robots that statically load an elastic mechanism, e.g. see [13], [14] and [15], which have the capability to perform very high jumps. However these robots present the drawbacks to require several seconds to load the jumping mechanism and, in the majority of the cases, they can not execute jumps at given instants.

The task, defined as in [16] for the PEA, is translated into the optimal control problem of maximizing the speed of the

The research leading to these results has received funding from the European Community's Seventh Framework Programme (FP7/2007-2013) under grant agreements n ICT-248587-2010 (THE) and ICT-287513(SAPHARI). Authors are with the Interdepart. Research Center "E. Piaggio", University of Pisa, Via Diotisalvi, 2, 56100 Pisa, Italy. Phone: +39 050 553639. Fax: +39050 550650. A. Bicchi is within the Department of Advanced Robotics, Istituto Italiano di Tecnologia, Via Morego, 30, 16163 Genova.

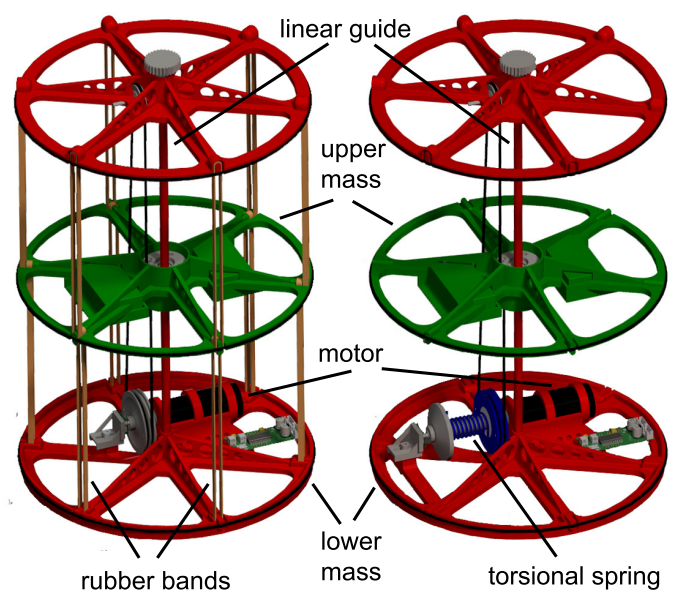

Fig. 1. A 3D view of the PEA and the SEA jumping robots under development; the logical scheme that represents the dynamic of these robots are reported in Fig. 2. The main components of the systems are: i) the lower mass (red structure) where the motor and its electronic control unit are located; its mass, that takes also into account the motor, electronic control unit and linear guide, is in the range of [350-450] [g]; ii) the upper mass (green part) that can slide longitudinally along the linear guide; its mass can be arbitrarily changed, from a minimum of 50 [g] to a maximum of 700 [g], putting on it additional weights; iii) the motor, which is a 10 [W] 24 [V] Maxon DC motor, has a reduction ratio of $4.4: 1$ for the PEA system and 12:1 for the SEA system and exploits the pulley to act the green mass by generating a force in both longitudinal directions. It has a stall torque of $64.6[\mathrm{mNm}]$ and no load speed of 9350 [rpm]. The pulley radius is $0.02[\mathrm{~m}]$; iv) the rubber bands represent the elastic elements; we use these elements because we can change the stiffness of the spring simply by substituting the rubber bands or varying their number.The length of the linear shaft is 0.35 $[\mathrm{m}]$; this allows us to obtain a stroke of $0.2[\mathrm{~m}]$ : it represents an additional constraint for the green mass motion.

upper mass of the two models at a predefined terminal time. In our work the optimization is subject to the following more realistic constraints: maximum elongation of the spring, contact condition between the environment and the robot, and torque-speed linear characteristic of the motor; while in [16] the constraints of the problem are only contact condition between the environment and the robot and motor torque. The solutions for the SEA and the PEA, have been obtained translating the optimal control problem into a convex programming problem which is later solved through a numerical tool. Later on, some constraints are relaxed in order to find the analytical solution for the robot with a parallel elastic actuator, considering a fixed final time and a known switching sequence of control but with unknown switching times.

In agreement with previous works (e.g. [17], [3], and [4]), section $\mathrm{V}$ of this paper shows the existence of an optimal stiffness of the elastic element which maximizes the performance of the soft actuators (both the SEA and the PEA) through the analysis of the dependency of the performance index on the parameters of the problem. Moreover, 
it is shown that the optimal stiffness depends on the task parameters and on the dynamic parameters of the system. These results suggest that the possibility to select the optimal stiffness, through a properly designed variable stiffness transmission, can further improve the performance provided that the overall mass of the system is not significantly increased.

Afterwards a comparison between the PEA and the SEA, given the power of the actuator but varying the transmission ratio, shows that the SEA with its optimal transmission ratio performs better then its PEA counterpart.

\section{DYNAMIC MODELS}

Let us consider a system of which the evolution can be described by two continuous phases, such as a jumping robot. The dynamic of this type of systems can be described by two continuous phases and a switching condition on the reaction exerted on the robot by the ground. The continuous phases are:

- the stance phase, with the contact between the lower mass and the environment. In this phase the normal force $R$, that the environment exerts on the robot, is positive and the distance between the lower part of the robot and the environment is zero;

- the flight phase, in which the contact is lost, $R=0$, and the distance between the robot and the environment is positive.

To formalize, let $t$ denotes the time and $T$ the instant time in which the robot looses contact, i.e. the terminal time of the stance phase. The following conditions are verified:

$$
\left\{\begin{array}{ll}
R>0 & \text { if } t \in[0, T) \\
R=0 & \text { if } t=T
\end{array} .\right.
$$

In the rest of the paper, we pay attention to two different linear jumping systems, Parallel Elastic Actuation (PEA) and Series Elastic Actuation (SEA):

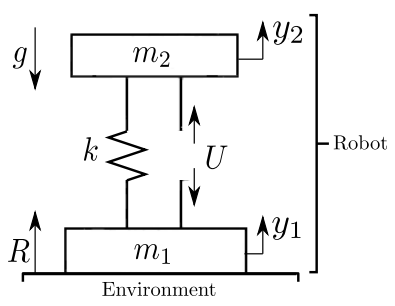

(a) PEA: it consists of two masses connected by a spring that has an actuator in parallel.

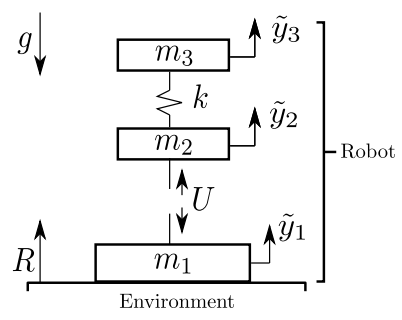

(b) SEA: it consists of three masses of which the two upper masses are connected by a spring and an actuator is interposed between the two lower masses.

Fig. 2. Model of PEA and SEA in touch with the environment.

\section{- PEA}

Referring to Fig. 2(a), we have that the dynamic of the system is described by the following equations:

$$
\left\{\begin{array}{l}
m_{1} \ddot{y}_{1}+k\left(y_{1}-y_{2}\right)=R-U-m_{1} g \\
m_{2} \ddot{y}_{2}+k\left(y_{2}-y_{1}\right)=U-m_{2} g
\end{array}\right.
$$

where $m_{1}$ and $m_{2}$ are the lower and upper mass inertia, $y_{1}$ and $y_{2}$ are the lower and upper mass position, $k$ is the stiffness of the spring, $U$ is the motor force, $R$ is the normal force and $g$ is the gravity force.
- SEA

Referring to Fig. 2(b), we have that the dynamic of the system is described by the following equations:

$$
\left\{\begin{array}{l}
m_{1} \ddot{\tilde{y}}_{1}=R-U-m_{1} g \\
m_{2} \ddot{\tilde{y}}_{2}+k\left(\tilde{y}_{2}-\tilde{y}_{3}\right)=U-m_{2} g \\
m_{3} \ddot{\tilde{y}}_{3}+k\left(\tilde{y}_{3}-\tilde{y}_{2}\right)=-m_{3} g
\end{array}\right.
$$

where $m_{1}, m_{2}$ and $m_{3}$ are the upper, intermediate and lower mass inertia, $\tilde{y}_{1}, \tilde{y}_{2}$ and $\tilde{y}_{3}$ are the upper, intermediate and lower mass position.

For both previous models, during the stance phase we impose the contact constraint between the robot and the environment while, at the instant $T$, the robot might jump. So we have that:

- in the range $0<t<T$, i.e. during the stance phase, the mass $m_{1}$ has to maintain contact with the environment, therefore its position and its velocity must be equal to zero

$$
\left\{\begin{array}{l}
y_{1}(t)=\tilde{y}_{1}(t)=0 \\
\dot{y}_{1}(t)=\dot{\tilde{y}}_{1}(t)=0
\end{array} \quad \forall t \in(0, T) ;\right.
$$

- at the instant $t=T$, that is the instant time of detachment, the mass $m_{1}$ must detach from the environment, therefore its position and its velocity must be positive

$$
\left\{\begin{array}{l}
y_{1}\left(T^{+}\right)=\tilde{y}_{1}\left(T^{+}\right)>0 \\
\dot{y}_{1}\left(T^{+}\right)=\dot{\tilde{y}}_{1}\left(T^{+}\right)>0
\end{array} .\right.
$$

We make the assumption to consider the initial conditions of the state of mass $m_{1}$ equal to zero

$$
\left\{\begin{array}{l}
y_{1}(0)=\tilde{y}_{1}(0)=0 \\
\dot{y}_{1}(0)=\dot{\tilde{y}}_{1}(0)=0
\end{array}\right.
$$

i.e. that the lower mass of robot is initially at rest and in contact with the environment.

Ultimately, by combining (4) and (6) we obtain

$$
\left\{\begin{array}{l}
y_{1}(t)=\tilde{y}_{1}(t)=0 \\
\dot{y}_{1}(t)=\dot{\tilde{y}}_{1}(t)=0
\end{array} \quad \forall t \in[0, T)\right.
$$

The consequence of (7) is that, during the stance phase $(\forall t \in[0, T))$, the state space vector may be reduced as shown below.

\section{A. PEA State Space Model}

Introducing the state vector $z=\left[\begin{array}{ll}z_{1} & z_{2}\end{array}\right]^{T}=\left[\begin{array}{ll}y_{2} & \dot{y}_{2}\end{array}\right]^{T}$, we have that (2) can be rewritten as

$$
\underbrace{\left[\begin{array}{c}
\dot{z}_{1} \\
\dot{z}_{2}
\end{array}\right]}_{\dot{z}}=\underbrace{\left[\begin{array}{cc}
0 & 1 \\
-\frac{k}{m_{2}} & 0
\end{array}\right]}_{A} \underbrace{\left[\begin{array}{c}
z_{1} \\
z_{2}
\end{array}\right]}_{z}+\underbrace{\left[\begin{array}{cc}
0 & 0 \\
\frac{1}{m_{2}} & -1
\end{array}\right]}_{B} \underbrace{\left[\begin{array}{c}
U \\
g
\end{array}\right]}_{u}
$$

and the constraints on the normal force (1) become

$$
\left\{\begin{array}{ll}
R=-k z_{1}(t)+U(t)+m_{1} g>0 & \text { if } t \in[0, T) \\
R=-k z_{1}(t)+U(t)+m_{1} g=0 & \text { if } t=T
\end{array} .\right.
$$




\section{B. SEA State Space Model}

Introducing the state vector $\tilde{z}=\left[\begin{array}{llll}\tilde{z}_{1} & \tilde{z}_{2} & \tilde{z}_{3} & \tilde{z}_{4}\end{array}\right]^{T}=$ $\left[\begin{array}{llll}\tilde{y}_{2} & \tilde{y}_{3} & \dot{\tilde{y}}_{2} & \dot{\tilde{y}}_{3}\end{array}\right]^{T}$, we have that (3) can be rewritten as

$$
\underbrace{\left[\begin{array}{c}
\dot{\tilde{z}}_{1} \\
\dot{\vec{z}}_{2} \\
\tilde{\widetilde{z}}_{3} \\
\tilde{z}_{4}
\end{array}\right]}_{\widetilde{z}}=\underbrace{\left[\begin{array}{cccc}
0 & 0 & 1 & 0 \\
0 & 0 & 0 & 1 \\
-\frac{k}{m_{2}} & \frac{k}{m_{2}} & 0 & 0 \\
\frac{k}{m_{3}} & -\frac{k}{m_{3}} & 0 & 0
\end{array}\right]}_{\widetilde{A}} \underbrace{\left[\begin{array}{c}
\widetilde{z}_{1} \\
\widetilde{z}_{2} \\
\widetilde{z}_{3} \\
\tilde{z}_{4}
\end{array}\right]}_{\widetilde{z}}+\underbrace{\left[\begin{array}{cc}
0 & 0 \\
0 & 0 \\
\frac{1}{m_{2}} & -1 \\
0 & -1
\end{array}\right]}_{\widetilde{B}} \underbrace{\left[\begin{array}{c}
U \\
g
\end{array}\right]}_{u}
$$

and the constraints on the normal force (1) become

$$
\left\{\begin{array}{ll}
R=U(t)+m_{1} g>0 & \text { if } t \in[0, T) \\
R=U(t)+m_{1} g=0 & \text { if } t=T
\end{array} .\right.
$$

\section{Problem Definition}

We are going to formulate the optimal jumping control problem to maximize the highest reachable point of the center of gravity of the robot, $h_{G, \max }$. Our optimization variable is the force exerted by the motor, $U$.

Since the take-off instant, only the gravity force acts on the robot; then by using the conservation of mechanical energy principle, we derive an estimation of $h_{G, \max }$ depending on the state of the robot soon after the take-off instant $T$ :

$$
h_{G, \max }=h_{G}\left(T^{+}\right)+\frac{V_{G}\left(T^{+}\right)^{2}}{2 g},
$$

where $h_{G}\left(T^{+}\right)$and $V_{G}\left(T^{+}\right)$are the height and the speed, respectively, of the center of gravity of the robot soon after the take-off instant. Due to the constraints on the upper mass displacement (Table I (i)) and (7), we can assume that the variation of $h_{G}(t)$ during the stance phase is negligible if compared to the variation of $V_{G}(t)^{2} /(2 g)$.

Hence we conclude that

$$
\max h_{G, \max } \approx \max V_{G}\left(T^{+}\right) .
$$

By the conservation of linear momentum principle, the speed of the robot soon after the take-off instant depends on the speed of the robot before the take-off instant:

$$
\begin{gathered}
V_{G}\left(T^{+}\right)=\frac{m_{2}}{m_{1}+m_{2}} z_{2}\left(T^{-}\right) \quad(\mathrm{PEA}) \\
V_{G}\left(T^{+}\right)=\frac{m_{3}}{m_{1}+m_{2}+m_{3}} \tilde{z}_{4}\left(T^{-}\right) \quad(\mathrm{SEA}) .
\end{gathered}
$$

It is remarked that, for the SEA, the intermediate mass models the inertia of the motor, and its magnitude is one order smaller than the other masses of the system. Then its contribution in the linear momentum equation is neglected to obtain the (15).

Hence, for both the SEA and the PEA, the performance index that will be used is the speed of the upper mass.

Table I summarizes the optimal jumping control problem that we formulate for the PEA and the SEA.

In the following we explain the meaning of the constraints reported in Table I.

Naturally we have to consider the system dynamic (Table I (b)) which represents a constraint in the maximization problem.

Further, in addition to the normal force constraints (Table I (c),(d)), in order to obtain realistic results we take into account also the torque-speed motor characteristic, so the force and the velocity that can be generated are bounded

\begin{tabular}{lll}
\hline PEA & SEA \\
\hline maximize $\quad z_{2}(T)$ & maximize $\widetilde{z}_{4}(T)$ & (a) \\
subject to & subject to \\
$\dot{z}(t)=A z(t)+B u(t)$ & $\dot{\tilde{z}}(t)=\widetilde{A} \widetilde{z}(t)+\widetilde{B} u(t)$ & (b) \\
$-k z_{1}(t)+U(t)+m_{1} g>0$ & $U(t)+m_{1} g>0$ & (c) \\
$-k z_{1}(T)+U(T)+m_{1} g=0$ & $U(T)+m_{1} g=0$ & (d) \\
$U_{\min } \leq U(t) \leq U_{\max }$ & $U_{\min } \leq U(t) \leq U_{\max }$ & (e) \\
$V_{\min } \leq z_{2}(t) \leq V_{\max }$ & $V_{\min } \leq \widetilde{z}_{3}(t) \leq V_{\max }$ & (f) \\
$V_{\min }-\frac{V_{\max }}{U_{\max }} U(t) \leq z_{2}(t)$ & $V_{\min }-\frac{V_{\max }}{U_{\max }} U(t) \leq \widetilde{z}_{3}(t)$ & (g) \\
$z_{2}(t) \leq V_{\max }-\frac{V_{\max }}{U_{\max }} U(t)$ & $\widetilde{z}_{3}(t) \leq V_{\max }-\frac{U_{\max }}{U_{\max }}$ & (h) \\
$\delta_{\min } \leq z_{1}(t) \leq \delta_{\max }$ & $\delta_{\min } \leq \widetilde{z}_{2}(t) \leq \delta_{\max }$ & (i) \\
& $\phi_{\min } \leq \widetilde{z}_{2}(t)-\widetilde{z}_{1}(t) \leq \phi_{\max }$ & (l) \\
\hline
\end{tabular}

TABLE I

PRoblem FORMULATION FOR THE PEA AND THE SEA: THE PERFORMANCE INDEX TO MAXIMIZE IS (a) AND ALL THE CONSTRAINTS ARE ((b)-(1)).

( $V_{\max }$ and $V_{\min }$ are the upper and lower bound for the speed of the actuator, $U_{\max }$ and $U_{\min }$ are the upper and lower bound for the force of the actuator) and coupled (Table I (e),(f),(g),(h)). In particular, during the stance phase, the motor speed $V(t)$ is equal to the velocity of the upper and intermediate mass for the PEA and the SEA, respectively, because the lower mass velocity is zero. An example of the simulation results that shows that the motor constraints are satisfied is shown in Fig. 3.

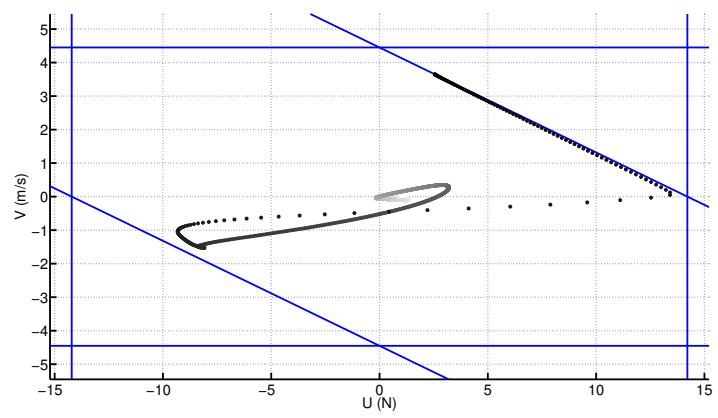

Fig. 3. The motor's constraints (blue) and the motor's work points (gray dotted) for one simulation: the points of the motor's work become darker and darker with the increase of time. All the constraints are satisfied.

It is also necessary to remark that:

- for the PEA, the constraint (Table I (i)) is the minimum between the stroke allowed by the robot and the maximum spring deformation $\left(\delta_{\max }\right.$ and $\delta_{\min }$ are the upper and lower bound for the position of the upper mass);

- for the SEA, we take into account both stroke (Table I (i)) and spring deformation (Table I (1)) constraints $\left(\delta_{\max }\right.$ and $\delta_{\min }$ are the upper and lower bound for the position of the upper mass, $\phi_{\max }$ and $\phi_{\min }$ are the upper and lower bound for the spring deflection). 


\section{Problem Solution}

In section IV-A we present the methodology that we use to find a numerical solution for the problems stated in Table I. Subsequently in section IV-B, we expose an approach that can be followed to determine an analytic solution for a simplified version of the optimal control problem: we apply it to the PEA case.

\section{A. Numerical Solution}

We used the notation for the PEA, but the results are valid also for the SEA, only substituting $z$ with $\tilde{z}$.

We used the notation for the PEA, but the results are also valid for the SEA, only substituting $z$ with $\tilde{z}$.

In order to numerically solve the two problems in Table I, we have discretized the state space dynamic equation using the forward Euler scheme:

$$
\dot{z}(t) \approx \frac{z(n+1)-z(n)}{\Delta t} .
$$

By using this transformation, the continuous-time system dynamic (Table I (b)) at the time instant $t=n$ becomes

$$
z(n+1)=(\Delta t A+I) z(n)+\Delta t B u(n),
$$

and the closed-form expression that allows us to determine the state of the system at a generic step $n$ w.r.t. the initial conditions is

$$
z(n)=(\Delta t A+I)^{n} z(0)+\sum_{i=0}^{n-1}(\Delta t A+I)^{(n-1-i)} \Delta t B u(i)
$$

Hence we can express the performance index and the constraints in Table I as a function of the initial state conditions $z(0)$ and of all the input control $u(i), i=0, \ldots, n-1$.

$T$ fixed and a sample time $\Delta t$ chosen, the number of samples is $N=T / \Delta t$. In particular, because $u=[U g]^{T}$ we consider as input control $U(i), i=0, \ldots, N-1$, so $U=$ $\left[\begin{array}{lll}U(0) & \ldots & U(N-1)\end{array}\right]^{T}$.

Let $C_{U, z_{i}}$ be the matrix of coefficients of $U$ associated with the $i$-th component of the state $z$, so

$$
C_{U, z_{i}}=\left[\begin{array}{cccc}
\Delta t B_{U, z_{i}} & 0 & \ldots & 0 \\
(\Delta t A+I)_{z_{i}} \Delta t B_{U, z_{i}} & \Delta t B_{U, z_{i}} & 0 & \vdots \\
\vdots & & \ddots & 0 \\
(\Delta t A+I)_{z_{i}}^{(n-1)} \Delta t B_{U, z_{i}} & (\Delta t A+I)_{z_{i}}^{(n-2)} \Delta t B_{U, z_{i}} & \ldots & \Delta t B_{U, z_{i}}
\end{array}\right],
$$

and let $C_{g, z_{i}}$ be the matrix of coefficients $g$ associated with the $i$-th component of the state $z$, defined in the same way as $C_{U, z_{i}}$.

Then, let $d_{z_{i}}$ the vector of the known terms associated with the $i$-th component of the state $z$, so

$$
d_{z_{i}}=z_{0, i}\left[\begin{array}{c}
(\Delta t A+I)_{z_{i}} \\
(\Delta t A+I)_{z_{i}}^{2} \\
\vdots \\
(\Delta t A+I)_{z_{i}}^{n}
\end{array}\right] .
$$

Using the above notation, we can express the state variables of the problems in Table $\mathrm{I}$ in function of the optimization variables $U$ :

$$
\left[z_{i}(1) z_{i}(2) \ldots z_{i}(N)\right]^{T}=C_{U, z_{i}} U+C_{g, z_{i}} g+d_{z_{i}} .
$$

Replacing (21) in (Table I (a)-(1)), we noticed that performance index and all the constraints are convex [18]. Hence we translated the optimal control problems into convex optimization problems.

Finally we used CVX [19] to solve the discrete convex optimization problems.

\section{B. Analytical Solution}

In this section, by exploiting the information coming from the numerical simulations, we derive an analytical solution for a problem similar to the ones reported in Table I: the motor is considered as a force source therefore the constraints on the velocity (Table I (f)) and (Table I (g)) are neglected.

According to (Table I (a)) the performance index depends only on the final state, in particular on the speed at terminal time.

W.r.t. the problems in Table I, we take into account only the constraints (Table I (b),(c),(e)). Because we have the inequality constraint on functions of the control and state variables, we need to use the augmented Hamiltonian [20] formulation:

$$
H(z(t), \lambda(t), U(t))=\lambda(t)^{T} \dot{z}(t)+\mu(t) C(z(t), U(t))
$$

where $\lambda(t)$ is the vector of the adjoint variables, $\mu:[0, T] \mapsto$ $\mathbb{R}$ satisfies the following condition

$$
\mu\left\{\begin{array}{ll}
=0 & \text { if } C<0 \\
\geq 0 & \text { if } C=0
\end{array},\right.
$$

and

$$
C(z(t), U(t))=-R
$$

with $R$ given by (9) for the PEA.

Since a constraint on both state and control variables is present we need to distinguish two different dynamic systems:

1) Constraint $C(z(t), U(t))$ is active (A.C.) In this case the optimal control value $U_{\text {opt }}(t)$ comes from the following equation

$$
C(z(t), U(t))=0
$$

and its value is reported in Table II. Substituting $U(t)=$ $U_{\text {opt }}(t)$ for the (A.C.) case in the state dynamic (8), we obtain

$$
\left\{\begin{array}{l}
\dot{z}_{1}(t)=z_{2}(t) \\
\dot{z}_{2}(t)=-\frac{m_{1}+m_{2}}{m_{2}} g \\
z_{1}\left(t_{0}\right)=c_{1}, \quad z_{2}\left(t_{0}\right)=c_{2}
\end{array}\right.
$$

while the value of $\mu(t)$ is given by the optimality necessary condition $\partial H / \partial U=0$ reported in Table II. By substituting the value of $\mu(t)$ in the co-state dynamic, whose expression is in Table II, we obtain

$$
\left\{\begin{array}{l}
\dot{\lambda}_{1}(t)=0 \\
\dot{\lambda}_{2}(t)=-\lambda_{1}(t)
\end{array} ;\right.
$$

2) constraint $C(z(t), U(t))$ is not active (N.A.C.)In this case

$$
\begin{aligned}
C(z(t), U(t)) & <0, \\
\mu(t) & =0,
\end{aligned}
$$

hence the state dynamic remains the one reported in (8)

$$
\left\{\begin{array}{l}
\dot{z_{1}}(t)=z_{2}(t) \\
\dot{z_{2}}(t)=-\frac{k}{m_{2}} z_{1}(t)+\frac{U(t)}{m_{2}}-g . \\
z_{1}\left(t_{0}\right)=c_{1}, \quad z_{2}\left(t_{0}\right)=c_{2}
\end{array} .\right.
$$


By substituting $\mu(t)=0$ in the co-state dynamic, whose expression is in Table II, we obtain

$$
\left\{\begin{array}{l}
\dot{\lambda}_{1}(t)=\frac{k}{m_{2}} \lambda_{2}(t) \\
\dot{\lambda}_{2}(t)=-\lambda_{1}(t)
\end{array} .\right.
$$

In the (N.A.C.) case, in agreement with the Pontryagin Maximum Principle, a necessary condition such that the optimal control maximizes the performance index is that it maximizes the Hamiltonian [20]. By analyzing the equation of the Hamiltonian, reported in Table II, and remembering that $\mu(t)=0$, we derive the optimal control law for the (N.A.C.), Table II.

\begin{tabular}{|c|c|}
\hline $\begin{array}{l}\text { Performance } \\
\text { index }\end{array}$ & $J=\left[\begin{array}{ll}0 & 1\end{array}\right]\left[\begin{array}{l}z_{1}(T) \\
z_{2}(T)\end{array}\right]$ \\
\hline Hamiltonian & $H=\lambda_{2}(t)\left[-\frac{k}{m_{2}} z_{1}(t)+\frac{U(t)}{m_{2}}-g\right]+\lambda_{1}(t) z_{2}(t)+\mu(t)\left[k z_{1}(t)-U(t)-m_{1} g\right]$ \\
\hline $\begin{array}{l}\text { Optimality } \\
\text { necessary } \\
\text { condition }\end{array}$ & $\frac{\partial H}{\partial U}=0 \Rightarrow \lambda_{2}(t) \frac{1}{m_{2}}-\mu(t)=0$ \\
\hline $\begin{array}{l}\text { Co-state } \\
\text { dynamic }\end{array}$ & $\left\{\begin{array}{l}\dot{\lambda}(t)=-\frac{\partial H}{\partial x}=\left[\begin{array}{cc}\frac{k}{m_{2}} \lambda_{2}(t)-\mu(t) k & -\lambda_{1}(t)\end{array}\right] \\
\lambda(T)=\frac{\partial z_{2}}{\partial z}=\left[\begin{array}{l}0 \\
1\end{array}\right]\end{array}\right.$ \\
\hline $\begin{array}{l}\text { State } \\
\text { (A.C.) }\end{array}$ & $\begin{array}{c}z_{1}(t)=\frac{-g\left(m_{1}+m_{2}\right)}{2 m_{2}} t^{2}+c_{1}+t c_{2} \\
z_{2}(t)=\frac{-g\left(m_{1}+m_{2}\right)}{m_{2}} t+c_{2}\end{array}$ \\
\hline $\begin{array}{l}\text { Co-State } \\
\text { (A.C.) }\end{array}$ & $\begin{array}{c}\lambda_{2}(t)=c_{\lambda, 1}+c_{\lambda, 2}(t-T) \\
\lambda_{1}(t)=-c_{\lambda, 2}\end{array}$ \\
\hline $\begin{array}{c}\text { State } \\
\text { (N.A.C.) }\end{array}$ & 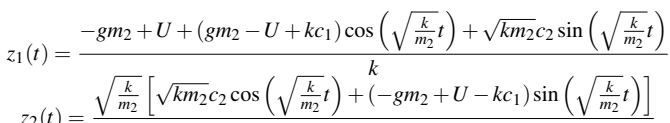 \\
\hline $\begin{array}{l}\text { Co-state } \\
\text { (N.A.C.) }\end{array}$ & $\begin{array}{c}\lambda_{2}(t)=c_{\lambda, 1} \cos \left(\sqrt{\frac{k}{m_{2}}}(t-T)\right)+c_{\lambda, 2} \sqrt{\frac{m_{2}}{k}} \sin \left(\sqrt{\frac{k}{m_{2}}}(t-T)\right) \\
\lambda_{1}(t)=\sqrt{\frac{k}{m_{2}}}\left[c_{\lambda, 1} \sin \left(\sqrt{\frac{k}{m_{2}}}(t-T)\right)-c_{\lambda, 1} \sqrt{\frac{m_{2}}{k}} \cos \left(\sqrt{\frac{k}{m_{2}}}(t-T)\right)\right]\end{array}$ \\
\hline $\begin{array}{l}\text { Optimal } \\
\text { control law }\end{array}$ & (N.A.C.) $\quad U_{\text {opt }}(t)=\left|U_{\text {lim }}\right| \operatorname{sgn}\left(\lambda_{2}(t)\right)$ \\
\hline
\end{tabular}

TABLE II

ANALYTICALLY SOLVED OPTIMAL CONTROL PROBLEM: THE SYMBOLS $c_{\lambda, l}, l=1,2$, DENOTE THE TERMINAL CONDITION FOR EACH INTERVAL OF THE CO-STATE DYNAMIC.

Assuming we know the optimal switching sequence by the numerical simulation but not the switching instants, in the following we show how to determine the time instants in which we have changes in the control input.

Let $t_{i}$ be the $i$-th switching instant and $\Delta t_{i}$ the $i$-th time interval, i.e. $\Delta t_{i}=t_{i}-t_{i-1}$. In order to find the solution of the optimal control problem, we exploit the closed-form integral of the dynamic of state and co-state reported in Table II: in particular

- we integrate backwards the co-state dynamic, until the initial time $t=0[\mathrm{~s}]$, using the known final conditions of the adjoint variable, $\lambda(T)$;

- we integrate forward the state dynamic, until the final time $t=T$, using the known initial conditions of the state variable, $z(0)$.
At the switching instants three different cases can occur:

1) if before and after the switching the dynamic follows the (N.A.C.) case, the condition below holds

$$
\lambda_{2}\left(t_{i}\right)=0 ;
$$

2) if in an interval in which the dynamic follows the (N.A.C.) we have that the constraint $C(z(t), U(t))$ becomes active and the following condition holds

$$
C\left(z\left(t_{i}\right), U\left(t_{i}\right)\right)=0 ;
$$

3 ) if the constraint $C(z(t), U(t))$ becomes active during a switching while the dynamic follows the (N.A.C.) case (this is the case of the second switching in Fig. 4), the following conditions hold

$$
\left\{\begin{array}{l}
C\left(z\left(t_{i}\right), U\left(t_{i}\right)\right)=0 \\
\lambda_{2}\left(t_{i}\right)=0
\end{array} .\right.
$$

Moreover, since this problem does not present pure state path constraint, we can exploit three further conditions for each switching time, i.e. the continuity of Hamiltonian, state and co-state (see [21]).

Finally we impose the fact that the final time is fixed, so we have

$$
\sum_{j} \Delta t_{j}=T
$$

By imposing all the previously described conditions, it is possible to derive a non-linear algebraic system of equations that allows us to obtain the switching times.

It is frequent that the values of the times can not be explicitly determined but, in authors' opinion, it is desirable to obtain an analytical even if implicit solution. In this case, one of the reason is that it allows us to evaluate quickly the optimal control policy and it could be decisive to perform real-time jumping tasks at given terminal times.

In the following we present an example that refers to the switching sequence of Fig. 4 with five intervals. The data that

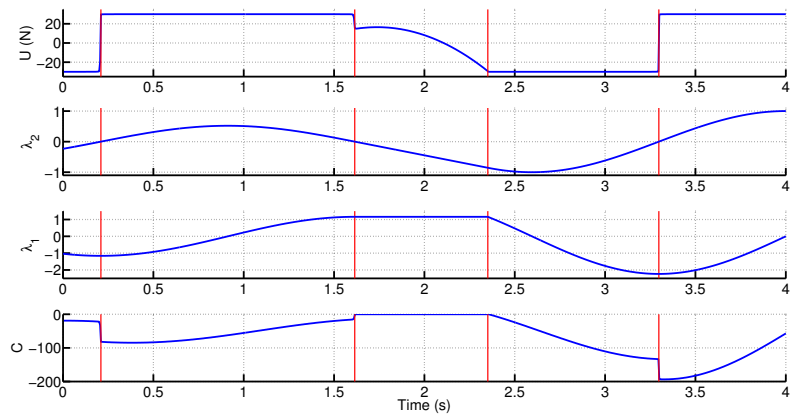

Fig. 4. Optimal control $U$, the adjoint variables $\lambda_{2}$ e $\lambda_{1}$, and the constraint $C$ for the solved problem: we highlighted the switching instants that we found by solving the analytical problems (red lines). When $\lambda_{2}$ cross zero and $C$ is not active then the control switches and the sign of the control is equal to the sign of $\lambda_{2}$.

we use to verify the solutions that we obtain are: $m_{1}=1$ $[\mathrm{kg}], m_{2}=4[\mathrm{~kg}], k=20[\mathrm{~N} / \mathrm{m}], U_{\max }=30[\mathrm{~N}], U_{\min }=$ $-30[\mathrm{~N}]$. The initial conditions for the state variables are the system's equilibrium: $z_{1_{0}}=-m_{1} g / k[\mathrm{~m}]$ and $z_{2_{0}}=0[\mathrm{~m} / \mathrm{s}]$. The terminal time is $T=4[\mathrm{~s}]$.

Considering for instance the switching sequence depicted in Fig. 4, we have that the last interval time is known because we know $\lambda_{2}(T)$, and $\lambda_{2}\left(t_{4}\right)=0$. Further, we also know $\Delta t_{2}$ 
because $\lambda_{2}\left(t_{2}\right)$ and $\lambda_{2}\left(t_{1}\right)$ are both equal to zero.

Hence we have only three unknown intervals. At this point we exploit the fact that the final time is fixed (31): we can express one of the three unknown intervals time in function of the other two, reducing the unknowns to two.

Finally, we impose the following two conditions that allow us to find the remaining unknowns: for the interval time where the constraint $C$ is active we have:

1) $\lambda_{2}\left(t_{2}\right)=0$;

2) $C\left(z\left(t_{3}\right), U\left(t_{3}\right)\right)=0$.

Combining these two algebraic equations we obtain a system of two equations in two unknown variables that allow us to determine all the instants of switching time, exploiting also the fact that the solutions must be real and positive.

Summing up what has been said, the values of the switching intervals can be determined by solving the following nonlinear algebraic system:

$$
\left\{\begin{array}{l}
\Delta t_{5}=\frac{\pi}{2 \omega} \\
\Delta t_{2}=\frac{\pi}{\omega} \\
\Delta t_{1}+\Delta t_{3}+\Delta t_{4}=T-\frac{3 \pi}{2 \omega} \\
k\left(-g \frac{m_{1}+m_{2}}{2 m_{1}} \Delta t_{3}{ }^{2}+\Delta t_{3} C_{1}\left(\Delta t_{1}\right)+C_{2}\left(\Delta t_{1}\right)\right)-U_{\min }-m_{2} g=0 \\
-\sin \left(\omega\left(T-\frac{3 \pi}{2 \omega}-\Delta t_{1}-\Delta t_{3}\right)\right)-\Delta t_{3} \omega \cos \left(\omega\left(T-\frac{3 \pi}{2 \omega}-\Delta t_{1}-\Delta t_{3}\right)\right)=0
\end{array}\right.
$$

where

$$
\begin{array}{r}
C_{1}\left(\Delta t_{1}\right):=-\frac{\omega^{2}}{k^{2}} \sin \left(\omega \Delta t_{1}\right) U_{\min } \sqrt{k m_{1}}, \\
C_{2}\left(\Delta t_{1}\right):=-2 \frac{g}{\omega^{2}}-\frac{g m_{1}}{k}+\frac{U_{\min }}{k}\left(1-\cos \left(\omega \Delta t_{1}\right)\right),
\end{array}
$$

with $\omega=\sqrt{k / m_{2}}$.

Using the switching times that we have found from the analytical solution, we verify that the trend of the simulations data matches with the trend of the data that we reconstruct by the dynamic equation of the state and co-state for the (A.C) and (N.A.C.) in Table II. We prove that:

- when the constraint $C$ is not active, the input control is bang-bang and it exactly switches when $\lambda_{2}$ is equal to zero;

- when the constraint $C$ is not active, the sign of the control $U$ is defined by the sign of $\lambda_{2}$.

\section{Simulation Results}

For the simulations we used the following data: $0.35 \leq$ $m_{1} \leq 0.45[\mathrm{~kg}], 0.1 \leq m_{2, P E A}=m_{3} \leq 0.8[\mathrm{~kg}], m_{2, S E A}=$ $1 / 11 m_{3}$ since it is considered that the inertia of the motor is negligible w.r.t. the other inertias of the system, $50 \leq k \leq 800$ $[\mathrm{N} / \mathrm{m}],-\delta_{\min }=\delta_{\max }=0.1[\mathrm{~m}],-\phi_{\min }=\phi_{\max }=0.3[\mathrm{~m}]$, $\Delta t=0.0005[\mathrm{~s}], g=9.81\left[\mathrm{~m} / \mathrm{s}^{2}\right]$. Since in the simulations we varied also the transmission ratio $\tau$, with $110 \leq \tau \leq 800$, the motor bounds can be rewritten as

$-U_{\min }=U_{\max }=\tau \tau_{s}, \quad-V_{\min }=V_{\max }=\frac{\Omega_{0}}{\tau}$,

where $\tau_{s}=64.6[\mathrm{mNm}]$ is the stall torque of the motor and $\Omega_{0}=9350[\mathrm{rpm}]$ is the no load speed of the motor.

A practical implementation of such values of transmission ratio can be obtained with a pulley of radius $0.02[\mathrm{~m}]$ and a gearbox with reduction ratio within the range $2.2 \div 16$.
The value of sampling time is two order of magnitude lower then the minimum time constant $t_{\min } \approx 0.36$ [s] of the systems under analysis.

In the following we present a discussion on the performance index dependance w.r.t the parameters of the systems. Terminal Time $T$ : the performance index increases according to the terminal time until a threshold value, after which the performance index reaches an upper bound (see Fig. 5 and Fig. 6). For both the PEA and the SEA we noticed
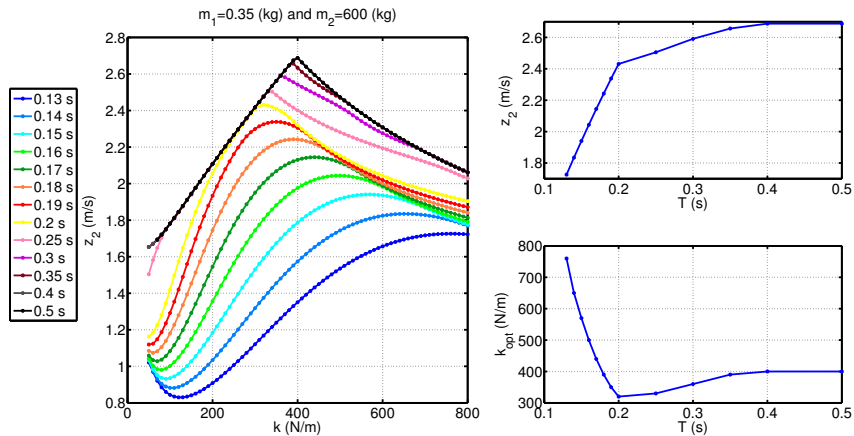

Fig. 5. Trends of the speed of the upper mass of the PEA w.r.t. variation on the final time $T$. It is possible to notice that exists a final time $\bar{T}$ at which the performance index does not change with the increase of the time.
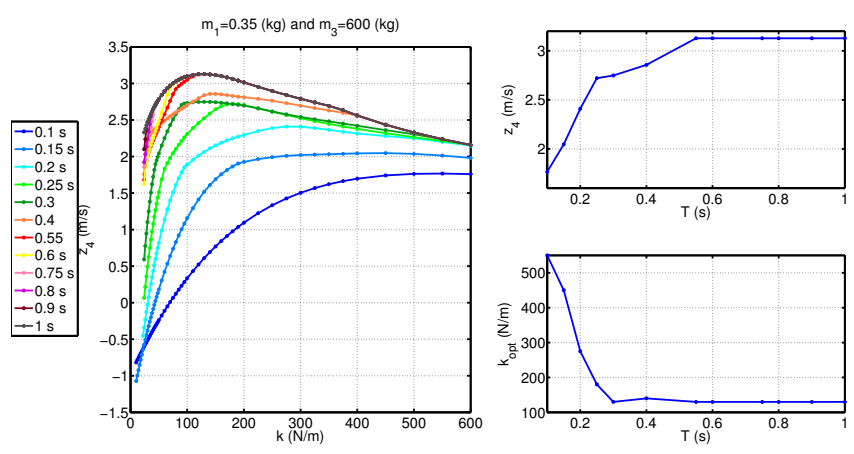

Fig. 6. Trends of the speed of the upper mass of the SEA w.r.t. variation on the final time $T$. It is possible to notice that exists a final time $\bar{T}$ at which the performance index does not change with the increase of the time.

that: (i) a final time $\bar{T}$ exists at which the performance index does not change with an increase of the time; (ii) the optimal stiffness decreases according to the increase of the terminal time.

Robot Upper Mass: given the power of the motor and the masses of the system, the constant stiffness that maximizes the performance varied (see Fig. 7).

We could summarize the data in Fig. 7 highlighting the optimal stiffness (see Fig. 8) and the optimal height (see Fig. 9) for each couple of upper and lower mass. For both the PEA and the SEA we noticed that: (i) the value of the optimal stiffness increases according to te increase of the upper mass; (ii) given the lower mass there exists an optimal upper mass that maximizes the performance.

Transmission Ratio $\tau$ : we performed simulations to obtain the optimal configuration for each gearbox transmission ratio in terms of upper mass and stiffness for both the SEA and the PEA. The results in Fig. 10 and in Fig. 11 show that: (i) there exists an optimal transmission ratio that maximizes the performance index; (ii) for the PEA the 

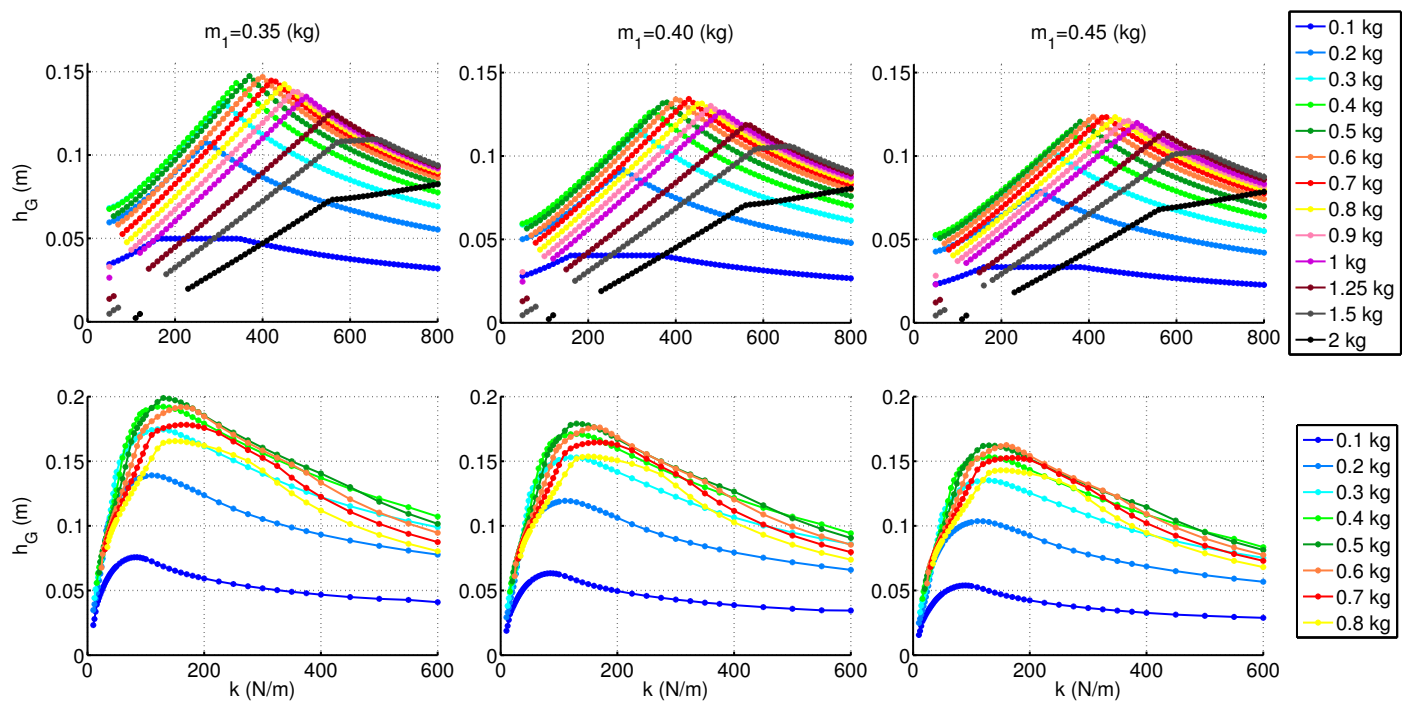

Fig. 7. Height of the center of gravity w.r.t. the stiffness. For each graph we kept constant $m_{1}$ and we change the value of $m_{2}$ and $m_{3}$ for the PEA (on the top) and for the SEA (on the bottom) respectively.
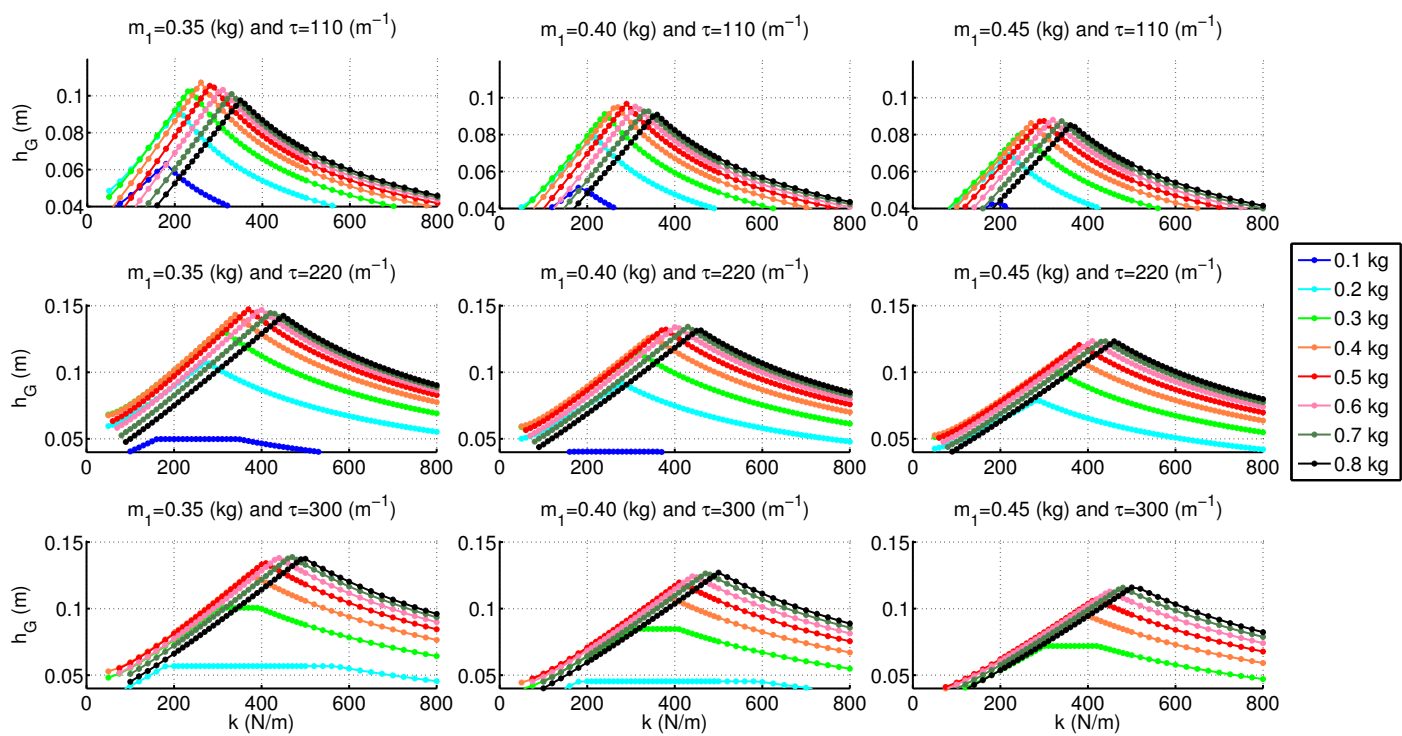

Fig. 10. Values of the jump height in the PEA system for different simulations performed with three different values of mass $m_{1}$ and three different values of transmission ratio. It is possible to notice that there is an optimal value of reduction ratio for which the maximum height jump is maximized.
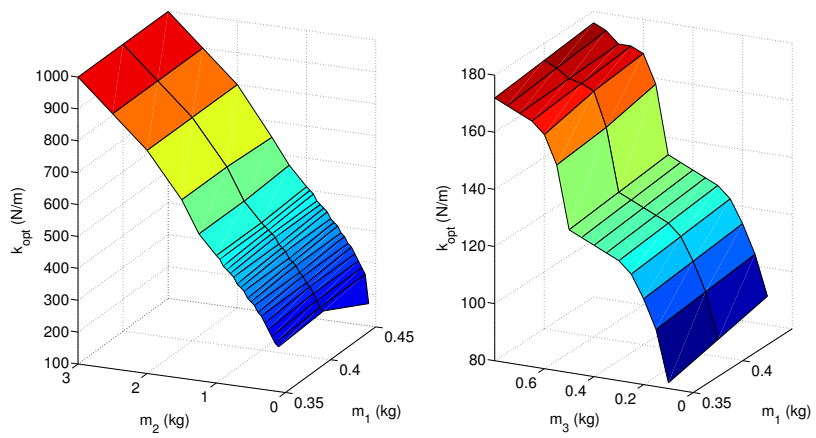

Fig. 8. Trend of the optimal stiffness for the PEA (on the left) and for the SEA (on the right) w.r.t. $\left(m_{1}, m_{2}\right)$ and $\left(m_{1}, m_{3}\right)$, respectively.
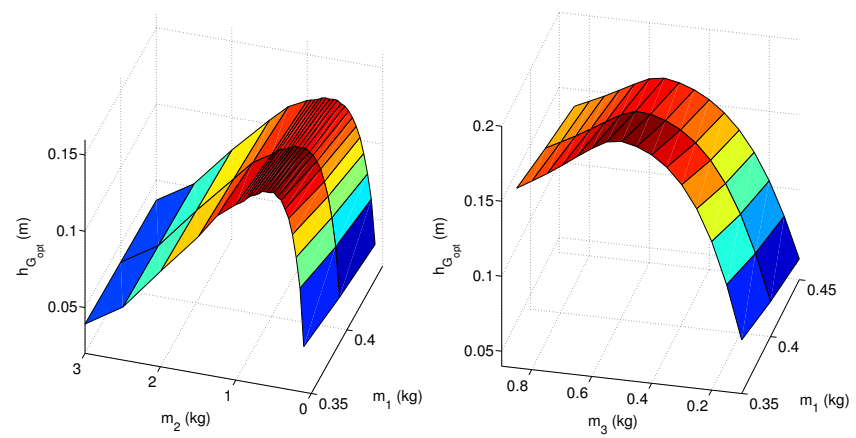

Fig. 9. Trend of the optimal height of the center of gravity for the PEA (on the left) and for the SEA (on the right) w.r.t. $\left(m_{1}, m_{2}\right)$ and $\left(m_{1}, m_{3}\right)$, respectively. 

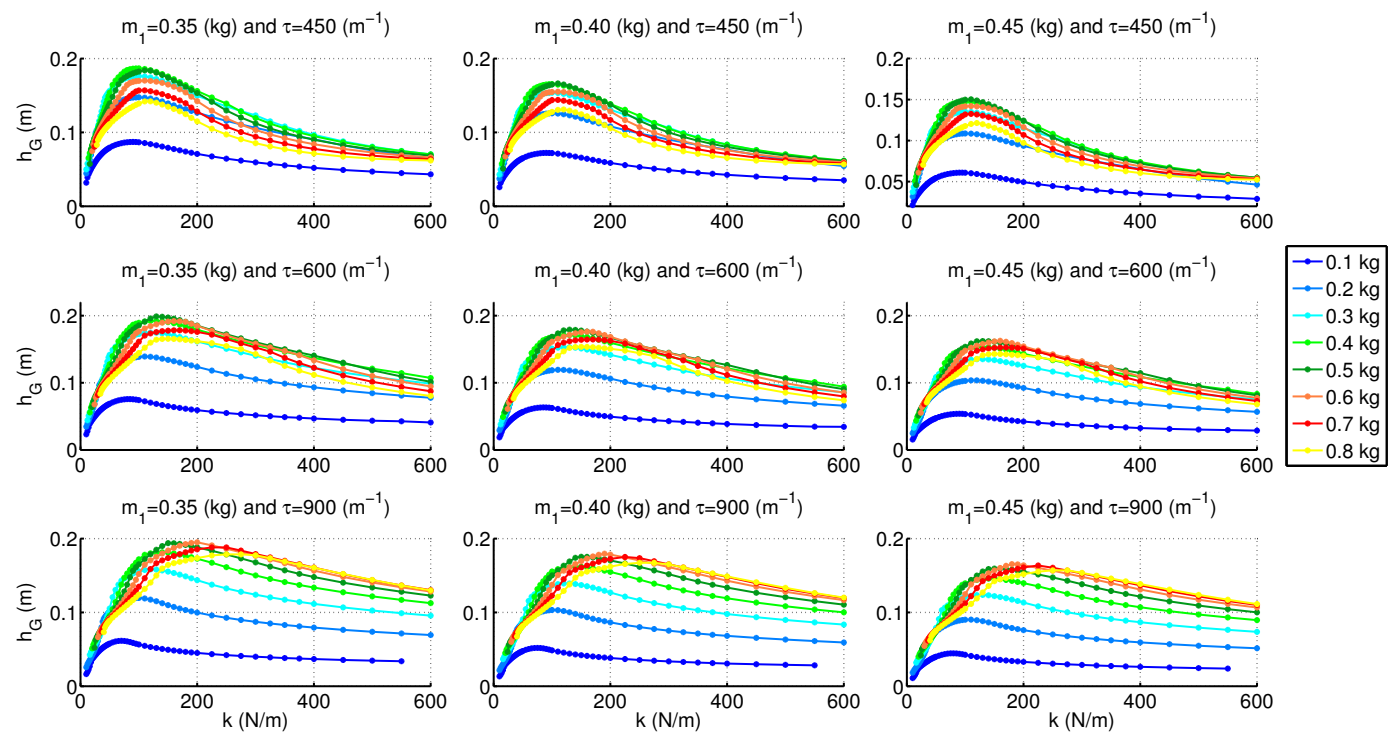

Fig. 11. Values of the jump height in the SEA system for different simulations performed with three different values of mass $m_{1}$ and three different values of transmission ratio. It is possible to notice that there is an optimal value of reduction ratio for which the maximum height jump is maximized.

optimal transmission ratio is low because the no load speed of the actuator is an upper bound for the speed of the upper mass; (iii) for the SEA the optimal transmission ratio is high because the speed limit of the motor does not constitute a direct upper bound for the speed of the upper mass.

\section{CONCLUSion ANd Future Work}

We compared the PEA and the SEA in the task of maximizing the jumping height. The results showed that: (i) given the power of the motor there exists an optimal constant stiffness; (ii) the optimal constant stiffness depends on terminal time, reduction ratio and inertial parameters; (iii) in the condition considered the SEA behaves better than the PEA. Comparing the optimal PEA and the optimal SEA configuration, we found that the latter performs better by about $25 \%$ (maximum PEA height $\approx 0.15[\mathrm{~m}]$ while maximum SEA height $\approx 0.2[\mathrm{~m}])$.

The results of this paper lead to think that the jumping task can be added to the other tasks (see [3], [2] and [17]) in which the exploitation of variable stiffness actuation can give decisive advantages w.r.t. rigid and constant elastic actuation. An open question is to study the behavior of nonlinear springs in such a task.

\section{ACKNOWLEDGMENTS}

The authors gratefully acknowledge the contribution of Lorenzo Malagia and Gustavo Incaini.

\section{REFERENCES}

[1] G. A. Pratt and M. Williamson, "Series elastic actuators," IEEE/RSJ Int. Conf. on Intelligent Robots and Systems, pp. 399-406, 1995.

[2] S. Haddadin, M. Weis, S. Wolf, and A. Albu-Schaffer, "Optimal control for maximmizing link velocity of robotic variable stiffness joints," IFAC World Congress Milan, August 2011.

[3] M. Garabini, A. Passaglia, F. Belo, P. Salaris, and A. Bicchi, "Optimality principles in variable stiffness control: The vsa hammer," in Intelligent Robots and Systems (IROS), 2011 IEEE/RSJ International Conference on, 2011.

[4] T. Hondo and I. Mizuuchi, "Analysis of the 1joint springmotor coupling system and optimization criteria focusing on the velocity increasing effect," IEEE ICRA, 2011.
[5] L. C. Visser, S. Stramigioli, and A. Bicchi, "Embodying desired behavior in variable stiffness actuators," in 2011 Congress of the International Federation of Automatic Control - IFAC 2011, Milano, italy, August 28 - September 2 2011, pp. 9733 - 9738.

[6] R. Ozawa and H. Kobayashi, "A new impedance control concept for elastic joint robots," in Robotics and Automation, 2003. Proceedings. ICRA 'O3. IEEE International Conference on, vol. 3, sept. 2003, pp. $3126-3131$ vol. 3

[7] M. Uemura, K. Kimura, and S. Kawamura, "Generation of energy saving motion for biped walking robot through resonance-based control method," in Intelligent Robots and Systems, 2009. IROS 2009. IEEE/RSJ International Conference on, oct. 2009, pp. 2928 -2933.

[8] J. Hurst and A. Rizzi, "Series compliance for an efficient running gait," Robotics Automation Magazine, IEEE, 2008.

[9] S. Cotton, I. Olaru, M. Bellman, T. van der Ven, J. Godowski, and J. Pratt, "Fastrunner: A fast, efficient and robust bipedal robot. concept and planar simulation," 2012.

[10] B. Vanderborght, B. Verrelst, R. V. Ham, M. V. Damme, D. Lefeber, B. Duran, and P. Beyl, "Exploiting natural dynamics to reduce energy consumption by controlling the compliance of soft actuators," Int Journal of Robotics Research, vol. 25, no. 4, pp. 343-358, 2006.

[11] N. Tsagarakis, Z. Li, J. Saglia, and D. Caldwell, "The design of the lower body of the compliant humanoid robot "ccub";," in Robotics and Automation (ICRA), 2011 IEEE International Conference on, 2011.

[12] M. Grimmer, M. Eslamy, S. Gliech, and A. Seyfarth, "A comparison of parallel- and series elastic elements in an actuator for mimicking human ankle joint in walking and running," in Robotics and Automation (ICRA), 2012 IEEE International Conference on, 2012.

[13] J. Zhao, N. Xi, B. Gao, M. Mutka, and L. Xiao, "Development of a controllable and continuous jumping robot," in Robotics and Automation (ICRA), 2011 IEEE International Conference on, 2011.

[14] M. Kovac, M. Fuchs, A. Guignard, J.-C. Zufferey, and D. Floreano, "A miniature 7g jumping robot," in IEEE ICRA, 2008.

[15] U. Scarfogliero, C. Stefanini, and P. Dario, "A bioinspired concept for high efficiency locomotion in micro robots: the jumping robot grillo," in ICRA 2006. Proceedings 2006 IEEE International Conference on.

[16] T. Yokozawa, S. Hara, and M. Ishikawa, "Optimal control strategy for high jump based on complementarity modeling," in Decision and Control, 2000. Proceedings of the 39th IEEE Conference on, 2000.

[17] A. Bicchi and G. Tonietti, "Fast and soft arm tactics: Dealing with the safety-performance tradeoff in robot arms design and control," IEEE Robotics and Automation Magazine, vol. 11, no. 2, June 2004.

[18] S. P. Boyd and L. Vandenberghe, Convex Optimization. Cambridge University Press, 2004.

[19] M. C. Grant and S. P. Boyd, The CVX Users' Guide, CVX Research, Inc., http://cvxr.com/cvx, September 2012

[20] A. Bryson and Y. Ho, Applied Optimal Control. Taylor \& Francis, 1975

[21] H. J. Pesch, "Real-time computation of feedback controls for constrained optimal control problems," Optimal Control Applications and Methods, pp. 129-145, 1989. 